\title{
Global Stabilizability and Observability imply Semi-global Stabilizability by Output Feedback
}

\author{
Andrew Teel \\ Dept. of Electrical Engineering \\ University of Minnesota \\ 4-174 EE/CS Building \\ 200 Union St. SE \\ Minneapolis, MN 55455 \\ USA
}

\author{
Laurent Praly \\ Centre Automatique et Systèmes \\ École des Mines de Paris \\ 35 rue St Honoré \\ 77305 Fontainebleau cédex \\ FRANCE
}

\begin{abstract}
We show that smooth global (or even semi-global) stabilizability and uniform complete observability are sufficient properties to guarantee semi-global stabilizability by dynamic output feedback for continuous-time nonlinear systems.
\end{abstract}

Keywords: Output feedback, Semi-global stabilizability, Uniform complete observability.

\section{Notation :}

- Let $(0)$ denote the number of the differential equation :

$$
\dot{x}=f(x) .
$$

For any continuous function $V: A \rightarrow \mathbb{R}$, with $A \subset \mathbb{R}^{p}$, we denote by $\dot{V}_{(0)}$ the Lie derivative of $V$ along the field $f$ when it exists on $A$, i.e. :

$$
\dot{V}_{(0)}(x)=\lim _{t \rightarrow 0} \frac{1}{t}[V(x+t f(x))-V(x)] \quad \forall x \in A .
$$

When $V$ is continuously differentiable we have trivially :

$$
\dot{V}_{(0)}(x)=\frac{\partial V}{\partial x}(x) f(x) \text {. }
$$

- $|\cdot|$ denotes the Euclidean norm.

- A function $f: A \rightarrow \mathbb{R}_{+}$, with $A \subset \mathbb{R}^{p}$, is said to be proper on $A$ if :

$$
\lim _{x \rightarrow \partial A} f(x)=\infty
$$

where $\partial A$ denotes the boundary of the set $A$. Note that if $f$ is proper on $A$ then, with $0 \leq c_{1} \leq c_{2}$, $\left\{x: c_{1} \leq f(x) \leq c_{2}\right\}$ is a compact subset of $A$.

- A function $f: A \rightarrow \mathbb{R}_{+}$is said to be positive (negative) definite on $B$, a subset of $A$, if $f(x)$ is strictly positive (negative) for all $x$ in $B$. 


\section{Main Result and Discussion}

\subsection{Main Result}

It is well established that a sufficient condition for global stabilizability by dynamic output feedback for an equilibrium point of a linear time-invariant system is stabilizability + observability. For this particular problem, it turns out that the crucial feature of a linear system is the fact that, as long as the control is bounded, no finite escape time is possible. Indeed, it has been proved by Sontag in [11] that the same (type of) condition is also sufficient for nonlinear systems which have no finite escape time. Unfortunately, if the finite escape time phenomenon is possible, then stabilizability and observability are no longer sufficient to get global stability. This has been proved by Dayawansa in [7]. He has shown that the following system on $\mathbb{R}^{2}$ :

$$
\left\{\begin{aligned}
\dot{x}_{1} & =x_{2} \\
\dot{x}_{2} & =x_{2}^{n}+u \\
y & =x_{1}
\end{aligned}\right.
$$

with $u$ in $\mathbb{R}$ as control and $y$ in $\mathbb{R}$ as the only measurement, cannot be globally stabilized by a finite dimensional dynamic continuous controller when the integer $n$ is larger than or equal to 3 . The objective of this paper is to show that stabilizability + observability is a sufficient condition for semiglobal, instead of global, stabilization by dynamic output feedback. To make this statement precise we need to specify what is meant by semi-global stabilizability, stabilizability and observability.

Definition 1 (Semi-global stabilizability) An equilibrium $x^{\star}$ of a dynamical system with measurement $y$ and control $u$ is said to be semi-globally stabilizable by dynamic output feedback if, for each compact set $\mathcal{K}$ there exist a dynamic output feedback $u=\theta_{1}(y, \zeta), \dot{\zeta}=\theta_{2}(y, \zeta)$ and a compact set $\mathcal{K}_{\zeta}$ such that the equilibrium $(x, \zeta)=\left(x^{\star}, 0\right)$ of the closed loop system is asymptotically stable with basin of attraction containing $\mathcal{K} \times \mathcal{K}_{\zeta}$.

Definition 2 (Stabilizability) An equilibrium point $x^{\star}$ of a dynamical system :

$$
\dot{x}=f(x, u)
$$

with $f$ a smooth function, $x$ in $\mathbb{R}^{n}$ and $u$ in $\mathbb{R}$ is said to be stabilizable if there exists a smooth ${ }^{1}$ function $\bar{u}$ such that $x^{\star}$ is a globally asymptotically stable equilibrium point of :

$$
\dot{x}=f(x, \bar{u}(x)) .
$$

Definition 3 (Uniform complete observability) A dynamical system:

$$
\left\{\begin{aligned}
\dot{x} & =f(x, u) \\
y & =h(x)
\end{aligned}\right.
$$

\footnotetext{
${ }^{1}$ In fact, it is sufficient for $\bar{u}$ to be $C^{l_{u}+1}$ with $l_{u}$ defined in (13).
} 
with $f$ and $h$ smooth functions, $x$ in $\mathbb{R}^{n},(u, y)$ in $\mathbb{R}^{2}$, is said to be uniformly completely observable if there exist two integers $n_{y}$ and $n_{u}$ and a smooth function $\Phi$ such that, for each solution of :

$$
\left\{\begin{aligned}
\dot{x} & =f\left(x, u_{0}\right) \\
\dot{u}_{0} & =u_{1} \\
& \vdots \\
\dot{u}_{n_{u}} & =v,
\end{aligned}\right.
$$

we have, for all t where the solution makes sense,

$$
x(t)=\Phi\left(y(t), \ldots, y^{\left(n_{y}\right)}(t), u_{0}(t), \ldots, u_{n_{u}}(t)\right)
$$

where $y^{(i)}(t)$ denotes the ith time derivative of $y$ at time $t .^{2}$

Theorem 1 If $x^{\star}$ is a stabilizable equilibrium point of a uniformly completely observable system then $x^{\star}$ is semi-globally stabilizable by dynamic output feedback.

\subsection{Discussion}

In the existing literature of which we are aware, little attention has been paid to semi-global stabilization by output feedback. Many recent results have been devoted to the global stabilizability case. However, due to the counterexample (4), we know that some restrictions must be introduced. The first class of restrictions concerns the growth of the nonlinearities: some kind of global Lipschitz condition or some more involved condition such as in [15] is imposed. In [6,3], a second class of restrictions is imposed. These restrictions are of a more geometric nature and imply that the system is linear up to output injection. This last assumption has been slightly relaxed in [10]. For other types of restrictions, see also $[8,9]$.

Results more closely related to ours are :

1. the work of Tornambè [13], where the same uniform complete observability property is assumed and the stabilizability property is implied by a feedback linearizability assumption. Unfortunately the proposed controller involves a high gain and is such that the guaranteed basin of attraction may vanish while this gain goes to infinity.

2. the work of Khalil and Esfandiari [4], where the function $\Phi$ in (9) of the uniform complete observability assumption depends only on $y$ and its derivatives and, as for Tornambè, the stabilizability property is implied by feedback linearizability. In this case, semi-global stabilizability is established.

Our work extends these two results by combining, in the controller, the observability property (9) and the dynamic extension (8) of Tornambè [13] with the high gain observer (21) and the saturated state estimates (22) of Khalil and Esfandiari [4]. Also, as we shall see, our result is only one of the many possible applications of the tools for semi-global stabilization we have proposed in [12].

\footnotetext{
${ }^{2}$ If $u$ is not present in (9), we let $n_{u}=-1$ in the following.
} 


\section{Proof of Theorem 1}

Let the system under consideration be described by :

$$
\left\{\begin{aligned}
\dot{x} & =f(x, u) \\
y & =h(x)
\end{aligned}\right.
$$

with $f$ and $h$ smooth functions, $x$ in $\mathbb{R}^{n}$ and $(u, y)$ in $\mathbb{R}^{2}$. We choose the coordinates so that the equilibrium point $x^{\star}$ is the origin.

\subsection{Design of a dynamic state feedback}

By successive derivations with respect to time, we can write $y$ and its time derivatives in terms of $u$ and its time derivatives and $x$. More precisely, with $n_{y}$ given by the uniform complete observability assumption, there exists a smooth function $\varphi$ and an integer number $m_{u} \leq n_{y}$ such that, for all $t$ where the solution makes sense,

$$
y^{\left(n_{y}+1\right)}(t)=\varphi\left(x(t), u(t), \ldots, u^{\left(m_{u}\right)}(t)\right) .
$$

Then, following the idea of Tornambè [13], let us consider the problem of designing a state feedback stabilizing controller for the extended system $^{3}$ :

$$
\left\{\begin{aligned}
\dot{x} & =f\left(x, u_{0}\right) \\
\dot{u}_{0} & =u_{1} \\
& \vdots \\
\dot{u}_{l_{u}} & =v
\end{aligned}\right.
$$

where :

$$
l_{u}=\max \left\{n_{u}, m_{u}\right\} .
$$

The solution to this stabilization problem is now well known and relies on the "adding one integrator technique" (see [14,2] for example) $)^{4}$. It follows, from this technique and the stabilizability assumption, that we are guaranteed of the existence of a smooth state feedback $v\left(x, u_{0}, \ldots, u_{l_{u}}\right)$ so that the following dynamic state feedback :

$$
\left\{\begin{aligned}
\dot{u}_{0} & =u_{1} \\
& \vdots \\
\dot{u}_{l_{u}} & =v\left(x, u_{0}, \ldots, u_{l_{u}}\right) \\
u & =u_{0}
\end{aligned}\right.
$$

makes the origin a globally asymptotically stable equilibrium point of (10).

\footnotetext{
${ }^{3}$ Typically $n_{u}=m_{u}-1$ and the dimension of the dynamic extension could be reduced by 1 but at the price of more involved notation in the following.

${ }^{4}$ We note that only a semi-globally stabilizing controller for (12) is needed in the following. Thus it would be sufficient here to apply the semi-global backstepping tool [12, Lemma 2.3]. Further, from this tool, we realize that the global stabilizability assumption of definition 2 can be replaced by semi-global stabilizability.
} 
In the following, we denote by $z$ the extended state vector in $\mathbb{R}^{n} \times \mathbb{R}^{l_{u}+1}$, i.e.

$$
z=\left(x^{\top}, u_{0}, \ldots, u_{l_{u}}\right)^{\top}
$$

By applying a converse Lyapunov Theorem (see [5] for example), we know the existence of a $C^{1}$ function $V: \mathbb{R}^{\left(n+l_{u}+1\right)} \rightarrow \mathbb{R}_{+}$which is positive definite on $\left(\mathbb{R}^{\left(n+l_{u}+1\right)}\right) \backslash\{0\}$, proper on $\mathbb{R}^{\left(n+l_{u}+1\right)}$ and satisfies:

$$
\alpha_{1}(|z|) \leq V(z) \quad, \quad \dot{V}_{(10),(14)}=-W(z)
$$

where the function $\alpha_{1}: \mathbb{R}_{+} \rightarrow \mathbb{R}_{+}$is strictly increasing and onto and the function $W: \mathbb{R}^{\left(n+l_{u}+1\right)} \rightarrow$ $\mathbb{R}_{+}$is continuous and positive definite on $\mathbb{R}^{\left(n+l_{u}+1\right)} \backslash\{0\}$. Also, let $\mathcal{K}_{u}$ be any compact subset of $\mathbb{R}^{l_{u}+1}$ containing the initial condition $\left(u_{0}(0), \ldots, u_{l_{u}}(0)\right)$ which is at our disposal. If $\mathcal{K}$ is the compact subset of $\mathbb{R}^{n}$ given in the semi-global stabilization problem, we know the existence of a positive real number $c \geq 1$ such that :

$$
\mathcal{K} \times \mathcal{K}_{u} \subset\{z: V(z) \leq c\} .
$$

Let us finally define the compact subset of $\mathbb{R}^{\left(n+l_{u}+1\right)}$ :

$$
\Gamma \doteq\{z: V(z) \leq c+1\}
$$

\subsection{Design of a dynamic output feedback}

Since $x$ is not measured, the controller (14) cannot be implemented. But from (9) in the uniform complete observability condition, we know that an implementation can be obtained if the time derivatives of $y$ are available, the time derivatives of $u$ being given by (14). We remark, by denoting :

$$
y_{i}=y^{(i)} \text {, }
$$

that (11) can be rewritten as:

$$
\left\{\begin{array}{cl}
\dot{y}_{0} & =y_{1} \\
& \vdots \\
\dot{y}_{n_{y}-1} & =y_{n_{y}} \\
\dot{y}_{n_{y}} & =\varphi\left(x, u_{0}, \ldots, u_{m_{u}}\right) \\
y & =y_{0} .
\end{array}\right.
$$

So, following the idea of Khalil and Esfandiari [4], let us propose the following "observer" :

$$
\begin{cases}\dot{\hat{y}}_{0} & =\widehat{y}_{1}+L \ell_{0}\left(y-\widehat{y}_{0}\right) \\ & \vdots \\ \dot{\hat{y}}_{n_{y}-1} & =\widehat{y}_{n_{y}}+L^{n_{y}} \ell_{n_{y}-1}\left(y-\widehat{y}_{0}\right) \\ \dot{\hat{y}}_{n_{y}} & =L^{n_{y}+1} \ell_{n_{y}}\left(y-\widehat{y}_{0}\right)+\varphi\left(\widehat{x}, u_{0}, \ldots, u_{m_{u}}\right) \\ \widehat{x} & =\operatorname{sat}\left(\Phi\left(\widehat{y}_{0}, \ldots, \widehat{y}_{n_{y}}, u_{0}, \ldots, u_{n_{u}}\right)\right)\end{cases}
$$


where $L \geq 1$ is a real number to be made precise later, the $\ell_{i}$ 's are the coefficients of a Hurwitz polynomial and "sat" is the bounded and globally Lipschitz function defined as :

$$
\operatorname{sat}(\cdot)=\min \left\{1, \frac{x_{\max }}{|\cdot|}\right\}(\cdot)
$$

with $x_{\max }$ the maximum value of $|x|$ on the compact set $\Gamma$. The motivation for introducing "sat" is that we know a priori, from the data of the semi-global stabilization problem, that the state component $x$ is initialized in the compact set $\mathcal{K}$. In fact, as we shall see below, we shall be able to guarantee that $z$ evolves in the compact set $\Gamma$. Consequently, an estimation of $x$ with norm larger than $x_{\text {max }}$ does not make sense and should be disregarded. Khalil and Esfandiari have proposed, in [4], using the saturation function "sat" to disregard such values.

With the estimated derivatives of $y$ given by the observer (21), we may propose the following dynamic output feedback :

$$
\left\{\begin{aligned}
\dot{\hat{y}}_{0} & =\widehat{y}_{1}+L \ell_{0}\left(y-\widehat{y}_{0}\right) \\
& \vdots \\
\dot{\hat{y}}_{n_{y}-1} & =\widehat{y}_{n_{y}}+L^{n_{y}} \ell_{n_{y}-1}\left(y-\widehat{y}_{0}\right) \\
\dot{\hat{y}}_{n_{y}} & =L^{n_{y}+1} \ell_{n_{y}}\left(y-\widehat{y}_{0}\right)+\varphi\left(\widehat{x}, u_{0}, \ldots, u_{m_{u}}\right) \\
\dot{u}_{0} & =u_{1} \\
& \vdots \\
\dot{u}_{l_{u}} & =v\left(\widehat{x}, u_{0}, \ldots, u_{l_{u}}\right) \\
\widehat{x} & =\operatorname{sat}\left(\Phi\left(\widehat{y}_{0}, \ldots, \widehat{y}_{n_{y}}, u_{0}, \ldots, u_{n_{u}}\right)\right) \\
u & =u_{0}
\end{aligned}\right.
$$

Denoting by $\zeta$ the vector in $\mathbb{R}^{n_{y}+l_{u}+2}$ given by :

$$
\zeta=\left(\widehat{y}_{0}, \ldots, \widehat{y}_{n_{y}}, u_{0}, \ldots, u_{l_{u}}\right)
$$

and by $\mathcal{K}_{\zeta}$ any compact subset of $\mathbb{R}^{n_{y}+l_{u}+2}$ containing the initial condition

$$
\left(\widehat{y}_{0}(0), \ldots, \widehat{y}_{n_{y}}(0), u_{0}(0), \ldots, u_{l_{u}}(0)\right),
$$

we have defined a candidate output feedback as well as a candidate compact set for meeting the semiglobal stabilizability property. To prove that these candidates are appropriate, we follow the two step analysis used in [10] and in [12] which is very common in the adaptive control field. Namely, we first establish that, with an appropriate choice of $L$, the solution enters an arbitrarily small neighborhood of the origin in finite time. This is a semi-global practical stability property. The proof of semi-global stability is then completed by showing that the origin is locally asymptotically stable.

\subsection{Semi-global practical stability}

First note that, by successive derivations of $y=h(x)$ with respect to time, one can find functions $\varphi_{i}$ 's so that $y$ and its derivatives satisfy, with (19),

$$
y_{i}=\varphi_{i}(z) \quad i=0, \ldots, n_{y} .
$$


Therefore, with $e$ the vector in $\mathbb{R}^{n_{y}+1}$ whose components are defined by :

$$
e_{i}=L^{n_{y}-i}\left(y_{i}-\widehat{y}_{i}\right)=L^{n_{y}-i}\left(\varphi_{i}(z)-\widehat{y}_{i}\right),
$$

the dynamics of the closed loop system $(10),(23)$ are completely described by :

$$
\left\{\begin{array}{l}
\dot{z}=\phi_{1}(z, e) \\
\dot{e}=L A e+\phi_{2}(z, e)
\end{array}\right.
$$

where $A$ is the Hurwitz companion matrix of the $\ell_{i}$ 's and the functions $\phi_{1}$ and $\phi_{2}$ are given by :

$$
\phi_{1}(z, e)=\left(\begin{array}{c}
f\left(x, u_{0}\right) \\
u_{1} \\
\vdots \\
u_{l_{u}} \\
v\left(x+\Delta_{1}(z, e), u_{0}, \ldots, u_{l_{u}}\right)
\end{array}\right) \quad, \quad \phi_{2}(z, e)=\left(\begin{array}{c}
0 \\
\vdots \\
0 \\
\Delta_{2}(z, e)
\end{array}\right)
$$

with, using (15),

$$
\Delta_{1}(z, e)=\operatorname{sat}\left(\Phi\left(\varphi_{0}(z)-\frac{e_{0}}{L^{n_{y}}}, \ldots, \varphi_{n_{y}}(z)-e_{n_{y}}, u_{0}, \ldots, u_{n_{u}}\right)\right)-x
$$

$$
\begin{aligned}
& \Delta_{2}(z, e)= \\
& \quad \varphi\left(x, u_{0}, \ldots, u_{m_{u}}\right)-\varphi\left(\operatorname{sat}\left(\Phi\left(\varphi_{0}(z)-\frac{e_{0}}{L^{n_{y}}}, \ldots, \varphi_{n_{y}}(z)-\epsilon_{n_{y}}, u_{0}, \ldots, u_{n_{u}}\right)\right), u_{0}, \ldots, u_{m_{u}}\right) .
\end{aligned}
$$

Since the function "sat" is bounded, the set $\Gamma$ is compact and we have the identities, using the uniform complete observability condition (9),

$$
x=\Phi\left(\varphi_{0}(z), \ldots, \varphi_{n_{y}}(z), u_{0}, \ldots, u_{n_{u}}\right), \quad z=\left(x^{\top}, u_{0}, \ldots, u_{l_{u}}\right)^{\top},
$$

we obtain the existence of a positive real number $\beta_{1}$ and a bounded, continuous function $\gamma$ with $\gamma(0)=0$ both independent of $L$ and satisfying :

$$
\left.\begin{array}{rl}
\left|\phi_{1}(z, e)-\phi_{1}(z, 0)\right| & \leq \gamma(|e|) \\
\left|\phi_{2}(z, e)\right| & \leq \beta_{1}
\end{array}\right\} \quad \forall(z, e) \in \Gamma \times \mathbb{R}^{n_{y}+1}, \quad \forall L \geq 1 .
$$

Recall also, from (16) and the definitions of "sat" and $\Gamma$, that, for the system :

$$
\dot{z}=\phi_{1}(z, 0)
$$

we have :

$$
\dot{V}_{(33)}=-W(z) \quad \forall z \in \Gamma .
$$

From this we could conclude semi-global practical stability by applying [12, Lemma 2.4]. For the sake of completeness, we reproduce here the proof of this Lemma.

Let $P$ be the solution of the Lyapunov equation :

$$
A^{\top} P+P A=-I .
$$


With $c$ and $V$ given in the conclusion of section 2.1, we define the function :

$$
U_{1}(z, e)=c \frac{V(z)}{c+1-V(z)}+\mu(L) \frac{\ln \left(1+e^{\top} P e\right)}{\mu(L)+1-\ln \left(1+e^{\top} P e\right)}
$$

where $\mu(L)$ is the function given by :

$$
\mu(L)=\ln \left(1+\gamma_{e} \lambda_{\max }\{P\} L^{2 n_{y}}\right)
$$

with, using the notation $(24)^{5}$,

$$
\gamma_{e}=\sup _{(z, \zeta) \in \mathcal{K} \times \mathcal{K}_{\zeta}}\left\{\left|\left(\begin{array}{c}
\varphi_{0}(z)-\widehat{y}_{0} \\
\vdots \\
\varphi_{n_{y}}(z)-\widehat{y}_{n_{y}}
\end{array}\right)\right|^{2}\right\} .
$$

Note the following three facts:

Fact 1 :

$$
\lim _{L \rightarrow \infty} \frac{L}{\mu(L)^{4}} \rightarrow \infty \quad, \quad \exists L_{0} \geq 1: \frac{d}{d L}\left(\frac{L}{\mu(L)^{4}}\right) \geq 0 \quad \forall L \in\left[L_{0},+\infty\right) .
$$

Fact 2 :

$$
(z, \zeta) \in \mathcal{K} \times \mathcal{K}_{\zeta} \quad \Longrightarrow \quad U_{1}(z, e) \leq c^{2}+\mu(L)^{2}
$$

This means that the initial conditions are in the set $\left\{(z, e): U_{1}(z, e) \leq c^{2}+\mu(L)^{2}\right\}$.

Fact 3: By defining the set $A_{1}$ as

$$
A_{1}=\{z: V(z)<c+1\} \times\left\{e: \ln \left(1+e^{\top} P e\right)<\mu(L)+1\right\},
$$

we have that, for each $L \geq 1, U_{1}(z, e): A_{1} \rightarrow \mathbb{R}_{+}$is positive definite on $A_{1} \backslash\{0\}$ and proper on $A_{1}$.

Our next task is to prove that, for each strictly positive real number $\rho$ smaller than two ${ }^{6}$, we can find a strictly positive real number $L_{*}>0$ such that, for all $L \geq L_{*}, \dot{U}_{1(27)}(z, e)$ is negative definite on the subset $\Lambda_{L}$ of $A_{1}$ defined as :

$$
\Lambda_{L} \doteq\left\{(z, e): \rho \leq U_{1}(z, e) \leq c^{2}+\mu(L)^{2}\right\} .
$$

First we note that :

$$
U_{1}(z, e) \leq c^{2}+\mu(L)^{2} \Longrightarrow\left\{\begin{array}{l}
V(z) \leq(c+1) \frac{c^{2}+\mu(L)^{2}}{c^{2}+\mu(L)^{2}+c}, \\
\ln \left(1+e^{\top} P e\right) \leq(\mu(L)+1) \frac{c^{2}+\mu(L)^{2}}{c^{2}+\mu(L)^{2}+\mu(L)}, \\
\frac{c}{c+1} \leq \frac{c(c+1)}{(c+1-V(z))^{2}} \leq \frac{\left(c^{2}+\mu(L)^{2}+c\right)^{2}}{c(c+1)} \\
\frac{\mu(L)}{\mu(L)+1} \leq \frac{\mu(L)(\mu(L)+1)}{\left(\mu(L)+1-\ln \left(1+e^{\top} P e\right)\right)^{2}} \leq \frac{\left(c^{2}+\mu(L)^{2}+\mu(L)\right)^{2}}{\mu(L)(\mu(L)+1)}
\end{array}\right.
$$

\footnotetext{
${ }^{5}$ If needed, $\gamma_{e}$ is increased so that, for all $L \geq 1$, we have $\mu(L) \geq 1$.

${ }^{6} \rho \leq 2$ implies $\rho \leq c^{2}+\mu(L)^{2}$
} 
We have also :

$$
\dot{U}_{1(27)}=\frac{c(c+1)}{(c+1-V(z))^{2}} \dot{V}_{(27)}+\frac{\mu(L)(\mu(L)+1)}{\left(\mu(L)+1-\ln \left(1+e^{\top} P e\right)\right)^{2}} \overbrace{\ln \left(1+e^{\top} P e\right)}(27) .
$$

But, from (34) and the bounds in (32), we can write:

$$
\left.\begin{array}{c}
\dot{V}_{(27)} \leq-W(z)+\beta_{2} \gamma(|e|) \\
\overbrace{\ln \left(1+e^{\top} P e\right)}^{(27)} \leq \frac{1}{1+e^{\top} P e}\left\{-L|e|^{2}+2 \lambda_{\max }\{P\} \beta_{1}|e|\right\}
\end{array}\right\} \quad \forall(z, e) \in \Gamma \times \mathbb{R}^{n_{y}+1}
$$

where $\beta_{2}$ is a positive real number which bounds $\frac{\partial V}{\partial z}$ on the compact set $\Gamma$. So, we get :

$$
\begin{aligned}
\dot{U}_{1(27)} \leq \frac{c(c+1)}{(c+1-V(z))^{2}}\left\{-W(z)+\beta_{2} \gamma(|e|)\right\} & \\
& +\frac{\mu(L)(\mu(L)+1)}{\left(\mu(L)+1-\ln \left(1+e^{\top} P e\right)\right)^{2}} \frac{1}{1+e^{\top} P e}\left\{-L|e|^{2}+2 \lambda_{\max }\{P\} \beta_{1}|e|\right\} .
\end{aligned}
$$

Now fix $L_{1}$ so that $\mu\left(L_{1}\right)^{2}=c^{2}+c$. Such a positive real number $L_{1}$ exists because the function $\mu(\cdot)$ is onto $[0,+\infty)$. Then using the bounds (43), $c \geq 1$, and choosing $L \geq L_{1}$ we have:

$$
\frac{1}{2} \leq \frac{c(c+1)}{(c+1-V(z))^{2}} \leq 2 \mu(L)^{4} .
$$

Thus we can rewrite (46) as:

$$
\begin{aligned}
\dot{U}_{1(27) \leq} \leq & \frac{c(c+1)}{(c+1-V(z))^{2}} \\
& \times\left\{-W(z)+\beta_{2} \gamma(|e|)+\frac{\mu(L)(\mu(L)+1)}{\left(\mu(L)+1-\ln \left(1+e^{\top} P e\right)\right)^{2}} \frac{1}{1+e^{\top} P e}\left[-\frac{L}{2 \mu(L)^{4}}|e|^{2}+4 \lambda_{\max }\{P\} \beta_{1}|e|\right]\right\} .
\end{aligned}
$$

Since, from (43), $\frac{c(c+1)}{(c+1-V(z))^{2}}$ is positive and bounded away from zero, it suffices to consider the expression :

$$
E(z, e) \doteq-W(z)+\beta_{2} \gamma(|e|)+\frac{\mu(L)(\mu(L)+1)}{\left(\mu(L)+1-\ln \left(1+e^{\top} P e\right)\right)^{2}} \frac{1}{1+e^{\top} P e}\left[-\frac{L}{2 \mu(L)^{4}}|e|^{2}+4 \lambda_{\max }\{P\} \beta_{1}|e|\right] .
$$

We are interested in evaluating this expression on the set $\Lambda_{L}$ defined in (42). We do so by considering the two sets:

$$
\begin{aligned}
& \Lambda_{1} \doteq\left\{(z, e): V(z) \leq c+1,1<\ln \left(1+e^{\top} P e\right)<\mu(L)+1\right\} \\
& \Lambda_{0} \doteq\left\{(z, e): V(z) \leq c+1, \ln \left(1+e^{\top} P e\right) \leq 1\right\} \cap\left\{(z, e): \rho \leq \frac{c V(z)}{c+1-V(z)}+\ln \left(1+e^{\top} P e\right)\right\}
\end{aligned}
$$

after observing that :

$$
\Lambda_{L} \subset\left(\Lambda_{1} \cup \Lambda_{0}\right)
$$

since :

$$
\left\{\ln \left(1+e^{\top} P e\right) \leq 1, \rho \leq U_{1}(z, e)\right\} \quad \Longrightarrow \quad \rho \leq \frac{c V(z)}{c+1-V(z)}+\ln \left(1+e^{\top} P e\right)
$$


i) About the set $\Lambda_{1}$ : From (39), there exists a real number $L_{2,0} \geq L_{0}$ such that, for each $L \geq L_{2,0}$, we have

$$
(z, e) \in \Lambda_{1} \quad \Longrightarrow \quad-\frac{L}{4 \mu(L)^{4}}|e|+4 \lambda_{\max }\{P\} \beta_{1} \leq 0 .
$$

Therefore, with the positivity of $W$, the boundedness of $\gamma, \mu(L) \geq 1$ and (43), we have:

$$
(z, e) \in \Lambda_{1} \quad \Longrightarrow \quad E(z, e) \leq \beta_{2} \sup _{e \in \mathbb{R}^{n_{y}+1}}\{\gamma(|e|)\}-\frac{1}{8} \frac{L}{\mu(L)^{4}} \frac{1}{\lambda_{\max }\{P\}} \frac{\exp (1)-1}{\exp (1)} .
$$

So, from (39) again, there exists a real number $L_{2} \geq L_{0}$ such that, for each $L \geq L_{2}$,

$$
(z, e) \in \Lambda_{1} \quad \Longrightarrow \quad E(z, e)<0
$$

ii) About the set $\Lambda_{0}$ : We follow here the same lines as in the proof of [1, Theorem 2], although we could have applied [12, Lemma 2.1]. The set $\Lambda_{0}$ is independent of $L$ and a compact subset of $\mathbb{R}^{\left(n+l_{u}+1\right)} \times \mathbb{R}^{\left(n_{y}+1\right)}$ and we have:

$$
(z, e) \in \Lambda_{0} \quad \Longrightarrow \quad E(z, e) \leq-W(z)+\beta_{2} \gamma(|e|)+8 \lambda_{\max }\{P\} \beta_{1}|e|-\frac{L}{4 \mu(L)^{4}} \frac{|e|^{2}}{\exp (1)} .
$$

To obtain this, we have used:

$$
\left\{\mu(L) \geq 1, \ln \left(1+e^{\top} P e\right) \leq 1\right\} \quad \Longrightarrow \quad \frac{1}{2} \leq \frac{\mu(L)(\mu(L)+1)}{\left(\mu(L)+1-\ln \left(1+e^{\top} P e\right)\right)^{2}} \leq 2 .
$$

Also we remark that there exists a strictly positive real number $\epsilon$ such that we have :

$$
\begin{aligned}
& \left\{e=0, \rho \leq \frac{c V(z)}{c+1-V(z)}+\ln \left(1+e^{\top} P e\right)\right\} \quad \Longrightarrow \quad \frac{2 \rho}{3} \leq V(z), \\
& \Longrightarrow \quad 0<\epsilon \leq W(z) \text {, } \\
& \Longrightarrow \quad E(z, \epsilon) \leq-\epsilon \text {. }
\end{aligned}
$$

Hence, by continuity and compactness, there exists an open set $\mathcal{V}$ containing the set $\{(z, e): e=0\}$ and such that:

$$
(z, e) \in \mathcal{V} \cap \Lambda_{0} \quad \Longrightarrow \quad E(z, e) \leq-\frac{\epsilon}{2}
$$

Also, the set $\Lambda_{0} \backslash \mathcal{V}$ is compact and such that $|e|$ is bounded away from zero. Let :

$$
M \doteq \max _{\Lambda_{0} \backslash \mathcal{V}}\left\{-W(z)+\beta_{2} \gamma(|e|)+8 \lambda_{\max }\{P\} \beta_{1}|e|\right\}
$$

and

$$
m \doteq \min _{\Lambda_{0} \backslash \mathcal{V}}\left\{\frac{|e|^{2}}{4 \exp (1)}\right\}>0 .
$$

Since the function $\frac{\cdot}{\mu(\cdot)^{4}}$ is increasing on $\left[L_{0}, \infty\right)$ and tends to $\infty$, there exists a positive real number $L_{3} \geq L_{0}$ such that, for each $L \geq L_{3}$,

$$
(z, \epsilon) \in \Lambda_{0} \backslash \mathcal{V} \quad \Longrightarrow \quad E(z, \epsilon) \leq M-\frac{L}{\mu(L)^{4}} m \leq M-\frac{L_{3}}{\mu\left(L_{3}\right)^{4}} m \leq-\frac{\epsilon}{2}
$$

We then take $L_{*}=\max \left\{L_{1}, L_{2}, L_{3}\right\}$ and we have proved: 
Lemma 1 For each real number $\rho \in(0,2]$, we can find a strictly positive real number $L_{*}(\rho)$ such that, for all $L \in\left[L_{*}(\rho),+\infty\right)$,

$$
\rho \leq U_{1}(z, e) \leq c^{2}+\mu(L)^{2} \quad \Longrightarrow \quad \dot{U}_{1(27)}(z, e)<0 .
$$

Also, the initial conditions of the closed loop system (10),(23) which are in the set $\mathcal{K} \times \mathcal{K}_{\zeta}$ satisfy:

$$
U_{1}(z, e) \leq c^{2}+\mu(L)^{2} .
$$

\subsection{Local stability}

To study the local properties of the closed loop system (10),(23) or equivalently (27), we restrict our attention to the set:

$$
\Lambda_{\rho} \doteq\left\{(z, e): U_{1}(z, e) \leq 2 \rho\right\}
$$

where $\rho \leq 2$ is now fixed so that, for all $L$ in $\left[L_{*}(\rho),+\infty\right)$,

$$
(z, e) \in \Lambda_{\rho} \quad \Longrightarrow \quad \operatorname{sat}(\bar{x})=\bar{x}
$$

where :

$$
\bar{x}=\Phi\left(\varphi_{0}(z)-\frac{e_{0}}{L^{n_{y}}}, \ldots, \varphi_{n_{y}}(z)-e_{n_{y}}, u_{0}, \ldots, u_{n_{u}}\right) .
$$

Such a choice for $\rho$ is possible since, with (16), (36) and $c$ and $\mu(L)$ larger than 1 , we have, for all $L$ in $\left[L_{\star}(\rho),+\infty\right)$,

$$
U_{1}(z, e) \leq 2 \rho \quad \Longrightarrow \quad\left\{|z| \leq \alpha^{-1}(4 \rho), e^{\top} P e \leq \exp (4 \rho)-1\right\} .
$$

Also the function $\alpha$ is strictly increasing and onto $[0,+\infty)$, and the functions $\Phi$ and $\varphi_{i}$ are continuous and, from their definitions, zero at zero.

With (29), (30) and (31), we have also the existence of positive real numbers $\gamma_{1}$ and $\nu$, not depending on $L$, such that (compare with $(32)$ ) :

$$
\left.\begin{array}{rl}
\left|\phi_{1}(z, e)-\phi_{1}(z, 0)\right| & \leq \gamma_{1}|e| \\
\left|\phi_{2}(z, e)\right| & \leq \nu|e|
\end{array}\right\} \quad \forall(z, e) \in \Lambda_{\rho} .
$$

With these inequalities, we could conclude local asymptotic stability directly by applying [12, Lemma 2.5]. A more explicit proof is provided in the following.

The inequalities (71) imply that $\overbrace{\sqrt{e^{\top} P e}}^{\cdot(27)}$ is well defined on $\Lambda_{\rho}$ and :

$$
\left.\begin{array}{rl}
\dot{V}_{(27)} & \leq-W(z)+\beta_{2} \gamma_{1}|e| \\
\overbrace{\sqrt{e^{\top} P e}}(27) & \leq-\frac{L}{2} \frac{e^{\top} e}{\sqrt{e^{\top} P e}}+\sqrt{\lambda_{\max }\{P\}} \nu|e|
\end{array}\right\} \quad \forall(z, e) \in \Lambda_{\rho} .
$$

This leads us to introduce another Lyapunov function candidate. We let :

$$
U_{2}(z, e)=V(z)+\sqrt{e^{\top} P e} .
$$


Indeed, by defining $L_{4}$ as :

$$
L_{4}=\max \left\{L_{*}(\rho),\left[2 \sqrt{\lambda_{\max }\{P\}}\left(1+\beta_{2} \gamma_{1}\right)+2 \lambda_{\max }\{P\} \nu\right]\right\},
$$

we obtain, for all $L$ in $\left[L_{4},+\infty\right)$,

$$
(z, e) \in \Lambda_{\rho} \quad \Longrightarrow \quad \dot{U}_{2(27)} \leq-W(z)-|e| .
$$

With the properties of $U_{2}$ and $W$, this implies that the origin is a locally asymptotically stable equilibrium point of the closed loop system.

\subsection{Semi-global asymptotic stability}

To get the conclusion of our Theorem, it remains to connect our semi-global practical stability result with this local asymptotic stability. To do so, we consider the semi-global Lyapunov function candidate :

$$
U_{3}(z, e)=\sigma\left(U_{1}(z, e)\right) U_{1}(z, e)+\tau\left(1-\sigma\left(U_{1}(z, e)\right)\right) U_{2}(z, e) .
$$

where $\tau$ is a strictly positive real number to be made precise later and $\sigma: \mathbb{R}_{+} \rightarrow[0,1]$ is a $C^{1}$ function which satisfies:

$$
\begin{aligned}
\sigma(x) & =1 \text { if } \quad 2 \rho \leq x, \\
& =0 \text { if } x \leq \rho, \\
\sigma^{\prime}(x) & <0 \text { if } \rho<x<2 \rho,
\end{aligned}
$$

where $\sigma^{\prime}$ is the first derivative of $\sigma$. With Lemma 1 and (75), we have established for all $L$ in $\left[L_{4},+\infty\right)$ :

$$
0<U_{1}(z, e) \leq c^{2}+\mu(L)^{2} \quad \Longrightarrow \quad \dot{U}_{3(27)}<\sigma^{\prime}\left(U_{1}(z, e)\right) \dot{U}_{1(27)}\left[U_{1}(z, e)-\tau U_{2}(z, e)\right] .
$$

By denoting by $\Re$ the support of $\sigma^{\prime}$, i.e. :

$$
\Re \doteq\left\{(z, e): \rho \leq U_{1}(z, e) \leq 2 \rho\right\},
$$

the semi-global stabilizability property will be established if we can find $\tau$ strictly positive so that:

$$
U_{1}(z, e)-\tau U_{2}(z, e) \geq 0 \quad \forall(z, e) \in \Re .
$$

But the functions $U_{1}$ and $U_{2}$ being continuous and positive definite on $\Re$, such a $\tau$ exists and is given as :

$$
\tau=\min _{(z, e) \in \Re}\left\{\frac{U_{1}(z, e)}{U_{2}(z, e)}\right\}
$$

\section{Concluding remarks}

We have proved an existence result for semi-global output feedback stabilization. Our controller involves a high-gain observer and for this reason its practical interest is unclear. Our assumptions do not go beyond standard stabilizability and observability. However, for the time being, this observability has to be uniform with respect to the control. As far as the stabilizability is concerned, it can be extended to the case where the feedback $\bar{u}$ involved in the stabilizability assumption is 
time-varying and dynamic. This feedback can even be only semi-globally stabilizing. Indeed, using the tools in [12], we know that if (14) is smoothly, semi-globally stabilizable by state feedback then (8) is also semi-globally stabilizable by state feedback. Then the global Lyapunov function given in (16) is replaced by a Lyapunov function defined on the basin of attraction of the extended system (10),(14). The existence of such a Lyapunov function comes from the results in [5].

As we have mentioned, the result presented here is a direct application of the tools described in

[12] where both academic and more practical applications of this result can be found.

\section{References}

[1] A. Bacciotti. Linear feedback: the local and potentially global stabilization of cascade systems. In Proceedings of IFAC Nonlinear Control Systems Design Symposium, Bordeaux, pages 21-25, June 1992.

[2] C. I. Byrnes and A. Isidori. New results and examples in nonlinear feedback stabilization. Systems and Control Letters, 12:437-442, 1989.

[3] I. Kanellakopoulos, P. V. Kokotovic, and A. S. Morse. A toolkit for nonlinear feedback design. Systems and Control Letters, 18(2):83-92, 1992.

[4] H. K. Khalil and F. Esfandiari. Semi-global stabilization of a class of nonlinear systems using output feedback. In Proceedings of the 31st Conference on Decision and Control, pages 34233428, December 1992.

[5] J. Kurzweil. On the inversion of Lyapunov's second theorem on stability of motion. Am. math. Soc. Transl., Ser. 2, 24:19-77, 1956.

[6] R. Marino and P. Tomei. Dynamic output feedback linearization and global stabilization. Systems $\&$ Control Letters, $17((2)): 115-121,1991$.

[7] F. Mazenc, L. Praly, and W. P. Dayawansa. Examples and counterexamples on global stabilization by output feedback. In preparation.

[8] J-B. Pomet, R. M. Hirschorn, and W. A. Cebuhar. Dynamic output feedback regulation for a class of nonlinear systems. to appear in Mathematics of Control, Signals and Systems, 1992.

[9] L. Praly. Lyapunov design of a dynamic output feedback for systems linear in their unmeasured state components. In Proceedings of IFAC Nonlinear Control Systems Design Symposium, pages 31-36, June 1992.

[10] L. Praly and Z. P. Jiang. Stabilization by output feedback for systems with iss inverse dynamics. Systems and Control Letters, 1992. to appear.

[11] E. D. Sontag. Conditions for abstract nonlinear regulation. Information and Control, 51(2), November 1981.

[12] A. R. Teel and L. Praly. Tools for semi-global stabilization by partial state and output feedback. Submitted for publication in SIAM J. of Control and Optimization. November 1992. 
[13] A. Tornambè. Output feeback stabilization of a class of non-minimum phase nonlinear systems. Systems and Control Letters, 19:193-204, 1992.

[14] J. Tsinias. Sufficient Lyapunov-like conditions for stabilization. Math. Control Signals Systems, 2:343-357, 1989.

[15] J. Tsinias. A generalization of Vidyasagar's theorem on stabilizability using state detection. Systems and Control Letters, 17:37-42, 1991. 